\title{
La transformación digital de la docencia universitaria en comunicación durante la crisis de la COVID-19 en España: una aproximación desde la perspectiva del alumnado
}

\section{Digital transformation of university teaching in communication during the COVID-19 emergency in Spain: an approach from students' perspective}

Antonio J. Baladrón Pazos. Universidad Rey Juan Carlos. España.

antonio.baladron@urjc.es

[CV] (1) G

Beatriz Correyero Ruiz. Universidad Católica de Murcia. España.

bcorreyero@ucam.edu

$[\mathrm{CV}] \odot \mathrm{R}^{\mathrm{C}} \mathrm{O}$

Benjamín Manchado Pérez. Universidad Complutense de Madrid. España.

benjaman@ucm.es

$[\mathrm{CV}] \odot \mathrm{R}^{\mathrm{R}}$

Cómo citar este artículo / Referencia normalizada

Baladrón Pazos, A. J., Correyero Ruiz, B y Manchado Pérez, B. (2020). La transformación digital de la docencia universitaria en comunicación durante la crisis de la COVID-19 en España: una aproximación desde la perspectiva del alumnado. Revista Latina de Comunicación Social, 78, 265-287. https://www.doi.org/10.4185/RLCS-2020-1477

\section{RESUMEN}

Introducción: La crisis sanitaria de la COVID-19 (enfermedad producida por el coronavirus o SARS-COV-2) en el año 2020 conllevó la suspensión de la docencia presencial en las universidades españolas y evidenció las dificultades para avanzar en la transformación digital del mundo universitario. El objetivo de la investigación cuyos resultados se exponen aquí es aproximarse, desde la perspectiva del alumnado, al modo en que los estudios universitarios en comunicación en España adaptaron la docencia de presencial a virtual durante dicha crisis. Metodología: Se aplicó un cuestionario online autoadministrado sobre una muestra del alumnado de Facultades de Comunicación de todas las Comunidades Autónomas de España en las que se imparten estos estudios. Resultados: El 57\% de los estudiantes aprueba la gestión de sus centros de estudio, porcentaje superior en las universidades privadas. El aula virtual ha sido la plataforma tecnológica de referencia (en el 84,5\% de casos) y los principales recursos docentes utilizados han sido la subida de archivos con temario $(73,2 \%)$ y la realización de videoconferencias $(67,9 \%)$, siendo este último el 
mejor valorado por los alumnos pese a no ser el más empleado. Conclusiones: Las Facultades de Comunicación -en el contexto de la universidad española- han sabido potenciar los medios de los que disponían para la docencia virtual, si bien la pandemia ha puesto en evidencia las limitaciones tecnológicas de las universidades. A grandes rasgos y dada la urgencia de la transformación, se ha trasladado al mundo online los métodos docentes propios de la enseñanza presencial en lugar de desarrollar una docencia que aproveche todas las potencialidades de lo virtual. En la docencia virtual, en especial en situaciones anómalas como la vivida, es fundamental el papel del profesor no sólo como transmisor de conocimientos sino en su papel de atención al alumno.

PALABRAS CLAVE: universidad; coronavirus; COVID-19; España; nuevas tecnologías; ciencias de la comunicación; formación e-learning.

\begin{abstract}
Introduction: The COVID-19 (SARS-CoV-2 or coronavirus disease) health crisis declared in 2020 led to the suspension of ongoing in-person learning in Spanish universities and showed the difficulties to move forward in the digital transformation of the university world. The objective of the research whose results are presented is to approach, from students' perspective, to the way in which university studies in Communication in Spain adapted ongoing in-person learning to online learning during such emergency period. Methodology: A self-administered online questionnaire was completed by a sample of Communication Faculties students from all the Spanish regions in which these studies are offered. Results: 57\% of the students approve the management of the situation by their Faculties, resulting in a higher percentage in private universities compared to public ones. Virtual classroom has been the most recognized technological platform (accounting for $84.5 \%$ of the cases) while the main teaching resources have been File uploading (73.2\%) and Videoconferences $(67.9 \%)$, the latter being the most valued by students while not the most employed. Discussion and results: The Faculties of Communication -in the context of University education in Spain- have succeeded in deploying the existing resources to virtual learning, although the pandemic has exposed some technological limitations of the universities. Broadly speaking and given the urgency of the transformation, common methods used for in-person learning have been shifted to the online world, instead of developing a teaching methodology that could benefit from all the advantages of virtual learning. Specifically, in anomalous situations like this one, the role of the teacher has proved to be essential, not only in transmitting knowledge but also in giving students attention and support.
\end{abstract}

KEYWORDS: university; coronavirus; COVID-19; Spain; new technologies; communication science; e-learning teaching

\title{
CONTENIDO
}

1. Introducción. 2. Objetivos. 3. Metodología. 4. Resultados y discusión. 5. Conclusiones. 6. Bibliografía 7. Curriculum Vitae

\section{Introducción}

A finales de enero y principios de febrero de 2020 se detectan en España los dos primeros casos positivos de la enfermedad COVID-19; la primera víctima mortal se registra el 13 de febrero. Durante este mes se suceden los casos positivos en distintos lugares del territorio español que el 8 de marzo eran ya más de 500, cifra que fue creciendo exponencialmente día tras día (más de 1.200 el 9 de marzo y más de 30 muertes). La situación obliga al gobierno regional de la Comunidad de Madrid a anunciar el 9 de marzo la suspensión de las clases presenciales en todos los niveles educativos durante dos semanas, viéndose afectados cerca de millón y medio de estudiantes; como 
consecuencia, al día siguiente la Conferencia de Rectores de las Universidades Públicas Madrileñas acuerda secundar la suspensión. El 12 de marzo ya es el Gobierno estatal quien recomienda a todas las Comunidades Autónomas el cierre de los centros de enseñanza, dado que la educación es una competencia que en España está transferida a los gobiernos regionales; ese mismo día, las Comunidades Autónomas suspenden las clases presenciales en sus universidades, así como en el resto de niveles educativos, medida que afectó a unos 9,5 millones de estudiantes. La situación de emergencia sanitaria era tal que, al día siguiente, el presidente del Gobierno anuncia que a partir del 14 de marzo entraría en vigor el estado de alarma en todo el territorio español mediante el Real Decreto 463/2020, de 14 de marzo, por el que se declara el estado de alarma para la gestión de la situación de crisis sanitaria ocasionada por la COVID-19; el 15 de marzo eran cerca de 8.000 los positivos y cerca de 300 los fallecidos, motivo por el cual el Gobierno de España fuerza a los españoles a un confinamiento obligatorio, con la prohibición de salir de sus residencias. A partir de este momento, tras sucesivas prórrogas del estado de alarma, se fueron estableciendo medidas cada vez más restrictivas que implicaron la práctica paralización de la vida, no sólo social, sino también económica y empresarial del país.

En este contexto, cerrados los campus y sin visos de poder reiniciar su actividad académica presencial, las universidades se ven obligadas a adoptar medidas para reorientar la docencia en sus titulaciones y adaptarse al nuevo entorno de enseñanza online, con la finalidad de no paralizar la actividad académica ni sus programas formativos. A contrarreloj, instituciones públicas y privadas elaboran planes y ponen a punto herramientas para llevar a cabo la docencia en remoto por parte de su profesorado. Las primeras, desde su autonomía universitaria y coordinadas por la Conferencia de Rectores de las Universidades Españolas (CRUE), organismo que en un comunicado oficial el día de la entrada en vigor del estado de alarma destaca que:

Los equipos de gobierno de las universidades (...) están arbitrando las medidas posibles para reducir los riesgos en la salud individual y colectiva de la comunidad universitaria y trabajando por el mejor desarrollo de la docencia no presencial para que la formación curricular no se vea afectada (...) Y, por ello, es vital atender la actividad académica de forma no presencial (CRUE, 2020a).

Las universidades tuvieron que replanificar on line asignaturas y titulaciones a apenas dos meses de terminar el curso académico, complementar los planes de e-learning para el profesorado o reforzar su limitada infraestructura tecnológica, con aulas virtuales no siempre preparadas para reconvertir su docencia presencial en online debido al alto volumen de tráfico que esto generaba (CaberoAlmenara, 2020; Nuere y De Miguel, 2020; UNESCO, 2020c). A estas limitaciones había que añadir la dificultad entre algunos sectores del alumnado para contar con dispositivos o conocimientos suficientes para un acceso a Internet eficiente que les permitiera hacer un seguimiento completo del curso (Frente de Estudiantes, 2020; Fundación CYD, 2020; UNESCO, 2020b). En este sentido, la CRUE solicitó a las universidades españolas la realización de una serie de encuestas entre los estudiantes que le permitieran hacer una radiografía aproximada de la realidad académica y poder afrontar la finalización del curso académico. La primera consulta fue para conocer las necesidades del alumnado internacional (CRUE, 2020b), en la segunda, impulsada por del rector de la Universidad de Castilla La Mancha -responsable además de la sección de Asuntos Estudiantiles de la CRUE-, se preguntó a los estudiantes sobre el calendario académico y las fórmulas de evaluación y, en la tercera, se pretendía conocer los recursos tecnológicos del alumnado, es decir, el tipo de equipos de los que disponía (portátil, Tablet, teléfono móvil) y la conexión, (si es fibra, móvil o cualquier otra) (Espinar, 2020). Para reducir esta brecha digital, el Fondo Supera Covid, iniciativa del Banco Santander y la CRUE, consiguió en el mes de mayo un presupuesto de dos millones de euros para financiar la compra de 5.000 ordenadores y dotar de 15.000 soluciones de conectividad a 
internet y webcams a jóvenes universitarios de toda España que se encontraran en una situación socioeconómica desfavorable (CRUE, 2020c).

Con mayor o menor celeridad, las universidades fueron convirtiendo la docencia presencial en docencia en remoto, adoptando diferentes medidas de acuerdo con las directrices consensuadas con el Ministerio de Universidades del Gobierno de España. Se organizó una mesa de trabajo técnica con la participación del Ministerio de Universidades, representantes de las Comunidades Autónomas, de las Agencias de Evaluación de la Calidad, de la Conferencia de Rectores de las Universidades Españolas y del Consejo de Estudiantes Universitarios del Estado que elaboró un borrador con los criterios generales que, respetando la autonomía universitaria, deberían seguir las universidades públicas en España para la adaptación del curso 2019-2020 con motivo de la pandemia de la COVID-19; dicho documento es acordado en la reunión del 15 de abril de la Conferencia General de Política Universitaria. Según se indica en el mismo:

El objetivo común es que ningún estudiante pierda este curso por esta crisis, ni sufra un sobrecoste económico derivado de las medidas que se adopten en el terreno docente. En segundo lugar, que administraciones, universidades y agencias aúnen esfuerzos para garantizar la calidad académica de las enseñanzas que reciban los estudiantes en este final de curso 2019-2020. (Ministerio de Universidades, 2020, p. 1).

Y añaden: "se considera adecuada la participación de la comunidad universitaria: del profesorado, el estudiantado y el personal de administración y servicios, a la hora de concretar estos criterios generales en las especificidades de cada titulación y/o materia" (Ministerio de Universidades, 2020, p. 2).

Conforme a este documento, las universidades y sus centros respectivos, entre ellos las Facultades de Comunicación, avanzaron en la reestructuración de todas sus dinámicas docentes. El cambio afectó a todos los niveles: adaptación de guías docentes, herramientas de docencia online, pruebas de evaluación, prácticas curriculares, procesos de acreditación de titulaciones, defensa de trabajos fin de grado y fin de máster, hasta a la propia reordenación de los calendarios académicos acordada por cada institución universitaria. Esta situación extraordinaria obligó a una actualización en tiempo récord en la forma de enseñar la comunicación por parte del profesorado, que en esos meses tuvo que trabajar por su actualización formativa y por adaptarse a marchas forzadas al nuevo entorno de elearning; lo cual supuso un auténtico reto para los estudios universitarios de comunicación que están a punto de cumplir medio siglo de vida en España, donde actualmente se imparten 203 grados de comunicación en 67 universidades y 101 centros (Postigo y Recoder, 2018).

\subsection{Nuevas tecnologías y docencia en comunicación en España}

Los estudios realizados sobre el estado de integración de las TIC en la universidad española han aumentado en los últimos años (Cubeles y Riu, 2018; Esteves, et al., 2018; De Pablos et al., 2019; Hernández, 2019; Colomer et al., 2020; De Casas, Caldeiro y Havrankova, 2020; Guillén y Mayorga, 2020; Liesa-Orús et al., 2020; Llorent, Tallon y De las Heras, 2020; Mercader, 2020). Baelo y Cantón (2009) diferencian, por una parte, los informes sobre las TIC en la universidad española y, por otro, las investigaciones desarrolladas dentro de las propias universidades sobre aspectos concretos de la integración de las TIC en la educación superior. Se podría añadir un tercer grupo referido a los informes sobre el uso de las TIC en la docencia de una determinada disciplina, sin duda los menos abundantes. En este último caso, en lo que respecta al campo de la comunicación, destacan los trabajos de Santín-Durán (2009), Gómez-Escalonilla et al. (2011), Santín et al. (2011) y Aparici y García Matilla (2016). 
El Informe Universidad 2000 o Informe Bricall (Bricall, 2000) constituyó el punto de partida para el desarrollo de importantes reformas en la educación superior española y puso de manifiesto cómo en la práctica totalidad de las universidades españolas se habían iniciado acciones para promover las TIC dentro de sus estructuras, aunque aún se observaba una excesiva dependencia de los métodos didácticos tradicionales; recogía la necesidad de que cada universidad potenciase el uso combinado de educación presencial con la virtual (Consejo de Universidades, 2003). En la actualidad, los informes y estudios sobre las TIC en la educación superior española más relevantes son los tres promovidos por la Sectorial de Tecnologías de la Información y las Comunicaciones de la Conferencia de Rectores de Universidades Españolas (CRUE): UNIVERSITIC, el Informe sobre el estado en formación online y tecnologías educativas (FOLTE) y el Informe TIC 360 (Tendencias TIC).

En cuanto al informe UNIVERSITIC, que se publica con carácter bianual desde 2004 (Barro, 2004) se estructura en torno a dos ejes fundamentales: Descripción de las TI y Gestión de las TI, que agrupan los principales procesos de la actividad universitaria: enseñanza-aprendizaje, investigación, gestión universitaria y gestión de la información, formación y cultura TIC y gestión de las TIC (recursos, proyectos, servicios dirección, calidad y normativa y colaboración). Para cada uno de estos ejes se definen una serie de indicadores que permiten recabar información de primera mano sobre la penetración real, el uso y las necesidades existentes en materia TIC en la educación superior española. A falta de la publicación del informe de 2019, el último publicado corresponde al curso 2017-2018 (CRUE, 2017), y las principales conclusiones muestran una consolidación de la implantación de las nuevas tecnologías como apoyo a la docencia con tasas de crecimiento más reducidas debido a los buenos niveles que se tienen como punto de partida. Según los datos que proporciona el informe, hemos llegado a un punto en el que "no podemos hablar de forma separada de servicios e infraestructuras TI para la docencia presencial y la no presencial, dada la evolución favorable de la docencia presencial con una fuerte utilización de las tecnologías educativas, tanto el aula como fuera de ella" (CRUE, 2017, p. 18).

Así, en los últimos años resulta muy común encontrarnos con titulaciones, muchas de ellas de comunicación, que ofrecen un determinado programa, asignatura o curso con una combinación de tiempos cara a cara, en el aula, y otros tiempos de trabajo fuera del recinto académico, con el apoyo de las tecnologías a través de apis virtuales, sitios webs de las asignaturas o paquetes integrados de aplicaciones de Google, wikis o redes sociales. Estas mezclas y combinaciones de métodos y recursos, denominadas blended-learning (Coaten, 2003; Bartolomé et al., 2018; García-Areito, 2018), ya se venían realizando por parte de las primeras universidades a distancia con el apoyo de las tutorías presenciales, mucho antes de la llegada de las tecnologías digitales (García Aretio y Marín Ibáñez, 1998). Los servicios de soporte TIC más empleados son la docencia virtual (en el 100\% de las universidades), así como el soporte a aulas de informática de uso docente (98\% de las universidades) y la gestión de licencias software para docencia (98\% de las universidades). Aquellos servicios que presentan un menor grado de uso son el soporte a realización y corrección de exámenes (76\%), el soporte a la elaboración de contenidos docentes $(78 \%)$ y el soporte a aulas multimedia (86\%), aunque en todos ellos se obtienen valores superiores con relación al informe anterior (CRUE, 2017). En cuanto a las plataformas más empleadas para la docencia virtual figuran, en primer lugar, Moodle, seguida de Moodle más desarrollos propios, Blackboad Collaborate, Sakai y, por último, plataformas creadas ad hoc por las propias universidades. En lo que respecta a los soportes para la realización y corrección de exámenes, lidera la clasificación también Moodle, seguida de Dara, plataformas ad hoc, plugin de Moodle, Sakay y Exaplus (CRUE, 2017). 
Asimismo, el informe de la CRUE pone también de manifiesto que el uso de servicios relacionados con la docencia virtual está muy extendido, tanto entre el profesorado como entre los propios alumnos. Un número medio de 1.805 profesores por universidad emplean la plataforma de docencia virtual institucional, lo que supone un porcentaje medio del $90,55 \%$ del total de docentes de cada institución. Además, la aplicación de las nuevas tecnologías de la información y la comunicación (TIC) a la enseñanza tiene su máxima expresión en la formación e-learning. Las universidades del Sistema Universitario Español (SUE) ofertan 10,76 titulaciones de media de carácter no presencial, lo que supone un porcentaje medio del $7,35 \%$ de todas las titulaciones de dichas universidades (CRUE, 2017). Además, según los últimos datos del Ministerio de Educación (Hernández y Pérez, 2019), el total de alumnos matriculados en universidades españolas no presenciales fue de 245.421, lo que supone el $15,4 \%$ del total de alumnos.

La formación e-learning llegó a las Facultades de Comunicación hace catorce años. La presencia de asignaturas prácticas y uso de herramientas tecnológicas (programas de edición, maquetación, diseño, etc...) en los estudios enmarcados dentro de la comunicación social hacían difícil la adaptación de los planes de estudio de estas carreras a la enseñanza a distancia. De hecho, son estudios que la UNED, pionera en la enseñanza a distancia en España, todavía no ha puesto en marcha, a pesar de que en varias ocasiones se han anunciado tentativas de ofrecer la carrera de Periodismo (Aneca, 2005, p. 104). En el caso de España, la primera universidad en crear estudios de grado online en el ámbito de la comunicación fue la Universidad Rey Juan Carlos (URJC), que ofertó por primera vez en el curso 2006-2007 los estudios de Periodismo bajo la modalidad "blendedlearning"; al año siguiente la URJC amplió esta oferta con la titulación de Publicidad y RR.PP. (Gómez-Escalonilla, Satín-Durán y Mathieu, 2011). En 2009 iniciaron los estudios de comunicación en modalidad a distancia la Universitat Oberta de Cataluña (UOC), que lanzó el Grado en Comunicación y el Grado en Información y Documentación; la Universidad Internacional de la Rioja (UNIR), que ofertó el grado en Comunicación; y la Universidad a Distancia de Madrid (UDIMA) incorporando a su oferta educativa el grado en Periodismo. La última universidad online que pondrá en marcha una titulación oficial en comunicación en modalidad online, concretamente el Grado en Periodismo, en el curso 2020-2021 es la Universidad Isabel I.

\subsection{Nuevas tecnologías y docencia durante la pandemia}

A pesar de los buenos resultados que arrojan los últimos informes sobre las TIC en la universidad española, lo cierto es que, como ya se ha adelantado, la pandemia ha puesto de manifiesto las debilidades del sistema para adaptar la docencia presencial al online. La crisis desencadenada ha propiciado la exigencia del desarrollo de una nueva configuración de la educación superior en la que cada universidad debe potenciar el uso combinado de la educación presencial con la virtual mediante la utilización de las TIC. Una vez declarado el estado de alarma y la cancelación de las clases presenciales, las autoridades estatales y comunitarias sugirieron, como ya adelantamos, que la docencia continuara impartiéndose por vía telemática (Ministerio de Universidades, 2020, p. 1). Así, a finales de marzo, la conferencia de rectores (CRUE) y el Ministerio de Universidades presentaron la plataforma, "Conect@ados: la Universidad en casa", un portal creado por la Universidad Nacional de Educación a Distancia (UNED) y por la Universitat Oberta de Catalunya, con el objetivo de ayudar a los profesores y estudiantes en la transición a la enseñanza digital a distancia. En la presentación del portal se dice que la web contendría metodologías para dar clases, así como conferencias, consejos, materiales de divulgación y atención personalizada para el cuidado psicológico de quienes lo requieran; sin embargo, algunos autores han criticado a esta plataforma por ser un mero repositorio de recursos (Pardo y Cobo, 2020, p. 17) y enlaces a portales webs de las universidades donde éstas recopilan sus indicaciones para la adaptación al contexto del Covid-19. 
Durante la pandemia, las estrategias de las universidades españolas, incluidas las que imparten estudios de comunicación, para adaptarse a la nueva situación y proseguir la actividad docente han sido de lo más diversas, aunque todas cuentan con un denominador común: campus virtual, que hasta ese momento había sido un soporte tecnológico para la docencia presencial, se convirtió en la plataforma de docencia de referencia. Las universidades a distancia partieron con ventaja, puesto que ya estaban adaptadas para impartir sus programas exclusivamente en remoto, y tanto alumnos como docentes estaban habituados a interactuar por medio de la plataforma; el principal problema al que tenían que hacer frente era la sustitución de los exámenes, el único procedimiento estrictamente presencial.

En lo que respecta a la adaptación de las enseñanzas presenciales a la modalidad a distancia, aunque se ha podido constatar un gran número de buenas prácticas docentes y el buen hacer de muchos equipos de desarrolladores, la crisis sanitaria ha demostrado que durante el proceso de "enseñanza remota de emergencia" (Hodges et al., 2020) muchas universidades "han improvisado, llegando tarde o con una preparación insuficiente a esta adaptación, aplicando voluntarismo y replicando en la virtualidad modelos de aprendizaje que ya están siendo obsoletos en la presencialidad" (Pardo y Cobo, 2020, p. 8). Se han puesto de relieve la diferencia de recursos de la educación universitaria española y las carencias tecnológicas de la mayoría de los centros educativos de enseñanza superior, los cuales están aún en vías de desarrollo en cuanto a lo digital, ya que la total integración de las TIC en la educación superior se encuentra aún lejos del aprovechamiento integral de las posibilidades de las mismas. Prueba de ello son los datos del estudio realizado durante el estado de alarma entre docentes de todas las universidades de España por investigadores de la Facultad de Ciencias Humanas y Sociales de la Universidad Pontificia Comillas, cuyos resultados revelan que, durante el confinamiento, el $30 \%$ del profesorado universitario español impartió docencia no presencial todos los días (el 55\% varias veces a la semana) cuando el 60\% de docentes nunca había impartido clases online (Ibercampus, 2020). Este estudio también constata que al 10\% de los docentes le ha costado trabajo enseñar a distancia, por no contar con conocimientos suficientes sobre enseñanza online, o que el $72 \%$ del profesorado ha adquirido conocimientos con cursos de la propia universidad (el $56,5 \%$ también con ayuda de compañeros a través de redes). Todos los docentes consultados afirman que, en general, sus conocimientos del entorno docente online se han incrementado en más de un $50 \%$ respecto a lo que sabían antes y el $32 \%$ se considera suficientemente preparado para seguir impartiendo docencia no presencial tras este período. Estos datos pondrían de manifiesto que, en general, los profesores se han adaptado bien y rápido a las nuevas metodologías y que se han familiarizado sin problemas con herramientas como Moodle, Blackboard Collaborate o Teams, que han sido las más utilizadas, según dicho estudio.

Mientras tanto, los universitarios de todas las disciplinas se han quejado durante el confinamiento de la falta de comprensión por parte del personal docente, al que critican por no haber tenido en cuenta las condiciones particulares que cada estudiante tenía en su casa; los alumnos argumentaban que se presuponía que todos contaban con un ambiente adecuado para el estudio y un equipo de trabajo propio, cuando esto no era así. Para superar la brecha digital entre aquellos estudiantes que no tenían acceso a los recursos necesarios para el seguimiento de las clases telemáticas, muchas universidades españolas pusieron disposición de los alumnos servicios extraordinarios de préstamo de ordenadores portátiles, webcams y tarjetas SIM. Sin embargo, en el caso de alumnos que no disponían en sus lugares de residencia de acceso ilimitado a Internet, la situación se hacía especialmente complicada. Además, hay que tener en cuenta que en muchos hogares varios miembros de la familia estaban teletrabajando o estudiando a distancia a la vez y que no todos los hogares tienen una conexión a Internet que soporte el consumo de datos por parte de todos al mismo tiempo. Tanto para docentes como para estudiantes se produjo además el "colapso del contexto" (Boyd, 2002), algo que ocurre cuando las personas, la información y las normas de diferentes entornos se encuentran y superponen. 
El confinamiento ha sido una situación anómala y provisional, pero ha demostrado la importancia del hardware, el software y la conectividad para cualquier estrategia de expansión digital del campus. Como afirman Pardo y Cobo (2020, p. 51), "estos problemas de acceso a tecnologías y conectividad imitan la diferencia entre universidades con recursos y el resto con mayores limitaciones, e inevitablemente profundizan la desigualdad histórica entre ambos tipos de instituciones".

En cuestión de exámenes, el Ministro de Educación, Manuel Castells, instó a las universidades a que definieran pruebas no presenciales (González, Marco y Medina, 2020). En el marco de la Conferencia de Rectores de España (CRUE), se propuso que la nota final del estudiante se distribuyera entre diferentes actividades que se pudieran realizar a lo largo del semestre, disminuyendo el peso del examen final; es decir, que se extendiera la evaluación continua que pregona el Plan Bolonia. No obstante, cada universidad adoptó una estrategia distinta, aunque a rasgos generales la mayor parte de docentes optaron porque los estudiantes realizaran tareas asíncronas o una evaluación "tipo test" con un tiempo limitado de respuestas a fin de intentar evitar posibles plagios e intercambios de información. Además, los alumnos de algunos grados debían instalar programas para garantizar la validez de la prueba, con funciones como bloqueo de pantalla y vigilancia por cámara; la obligatoriedad de este sistema fue cuestionada por algunos estudiantes, que consideran que, al otorgar permisos para la grabación de imágenes y audio desde su dispositivo, podría verse comprometido su derecho a la privacidad.

En la actualidad, la mayoría de las universidades del mundo están modificando su planificación docente planteándose varios escenarios. Hay expertos que dicen que este movimiento hacia la virtualidad impuesto por la pandemia de la COVID-19 ha llegado para quedarse, mientras que otros apuestan por la puesta en valor de la enseñanza presencial combinada con experiencias de aprendizaje en remoto dentro del proceso integral de formación de los estudiantes. En cualquier caso, las TIC cobrarán un importante protagonismo en la era post-pandemia conformándose en algo más que una "muleta transitoria" (Chiape, 2016; Orihuela, 2020; Onyema et al., 2020) y siendo elementos esenciales para el desarrollo de la flexibilidad organizativa de las enseñanzas y el desarrollo de nuevas sinergias que conllevan plenamente a las universidades hacia las sociedades del conocimiento (Castells, 2001, 2006; Coll y Martí, 2001), todo ello en una dinámica de cambio y reflexión sobre el qué, el cómo y el para qué de la educación del siglo XXI (Castañeda y Selwyn, 2020, Orihuela, 2020; LeBlanc, 2020; Pardo y Cobo 2020).

\section{Objetivos}

Para abordar con rigor científico el objeto de estudio planteado, se desarrolló una investigación cuyo objetivo genérico era una aproximación, desde la perspectiva de los estudiantes, al modo en que los estudios universitarios en comunicación en España se adaptaron a la docencia virtual durante la crisis sanitaria de la COVID-19; interesaba conocer las percepciones del alumnado, por tanto superando lo investigado en otros estudios que se han realizado y que anteriormente han sido mencionados, centrados en los docentes. Este objetivo genérico se debía traducir en los siguientes objetivos específicos:

1. Conocer el grado de satisfacción que tienen los estudiantes de comunicación acerca de la adaptación de sus centros a la docencia virtual durante la suspensión de las clases presenciales.

2. Identificar, desde la perspectiva de los estudiantes, los principales recursos docentes y plataformas tecnológicas empleados en la docencia virtual de la comunicación durante la suspensión de las clases presenciales. 
3. Conocer la valoración para su aprendizaje que los estudiantes de comunicación hacen de dichos recursos docentes, así como de las diferentes tareas propuestas por los profesores en sus asignaturas durante la suspensión de las clases presenciales.

\section{Metodología}

Con este fin, se realizó una investigación descriptiva, utilizando como técnica cuantitativa un cuestionario online autoadministrado. Se elaboró a través de la aplicación online de formularios Google Forms permitiendo, de manera sencilla, la recogida y posterior volcado de datos para su análisis. Dada la confidencialidad en el tratamiento de los datos y su uso estrictamente para los objetivos de la investigación, en el encabezamiento se hacía constar el carácter anónimo de las respuestas aportadas, así como la utilización de los datos únicamente con fines académicos, de modo que se asumía un consentimiento implícito por la participación en la investigación sin que fuera necesaria una negativa expresa.

Tal y como se adjunta a continuación, el cuestionario estaba compuesto de ocho preguntas. En las dos primeras se intentaron identificar, de forma objetiva, los recursos y las plataformas empleados por el cuerpo docente para la enseñanza virtual, ofreciendo la posibilidad de marcar una o varias respuestas de entre un rango presentado (incluida la de "Ninguno"), a las que se podrían añadir aquellos ítems que el alumnado considerara. Las siguientes tres preguntas recogían una valoración subjetiva de la calidad de la docencia en su modalidad virtual (a través de una escala numérica), así como de los recursos empleados y las tareas llevadas a cabo en ese periodo (empleando, en estos casos, escalas ordinales de puntuación). De esta manera, con las cinco primeras preguntas se podrían abarcar los diversos aspectos que se planteaban como objetivos de la investigación. Por otro lado, las tres últimas correspondían a la identificación de los estudios de grado/posgrado, y de la institución en la que se encontraba estudiando: en concreto, información sobre el tipo de universidad, la titulación cursada y el nombre completo de la universidad (con un desplegable incluyendo todas las universidades españolas).

\section{Tabla 1. Cuestionario}

\begin{tabular}{|rl|}
\hline \multicolumn{1}{|c|}{ Cuestionario } \\
\hline 1. ¿Cuáles son los recursos que están utilizando tus profesores para la docencia virtual de \\
su asignatura? (señala los más usados hasta un máximo de 3) \\
- & Chat \\
- & Foro \\
- & Glosario \\
- & Mail \\
- & Subida de archivos (temario) \\
- & Tareas on line (prácticas) \\
- & Test de autoevaluación \\
- & Videoconferencia \\
- & Wiki \\
- & Ninguno \\
- & Otro [campo editable] \\
& \\
\hline ¿Qué & plataformas están utilizando tus profesores para la docencia virtual de su \\
asignatura? (señala las más usadas hasta un máximo de 3) \\
- $\quad$ Aula virtual de tu universidad \\
- $\quad$ Blackboard Collaborate \\
- $\quad$ Blogs y espacios web de la asignatura \\
- $\quad$ Microsoft Teams \\
- $\quad$ Redes sociales \\
- $\quad$ Skype \\
- $\quad$ Whatsapp \\
\hline
\end{tabular}


RLCS, Revista Latina de Comunicación Social, 78, 265-287

[Investigación] DOI: 10.4185/RLCS-2020-1477| ISSN 1138-5820| Año 2020

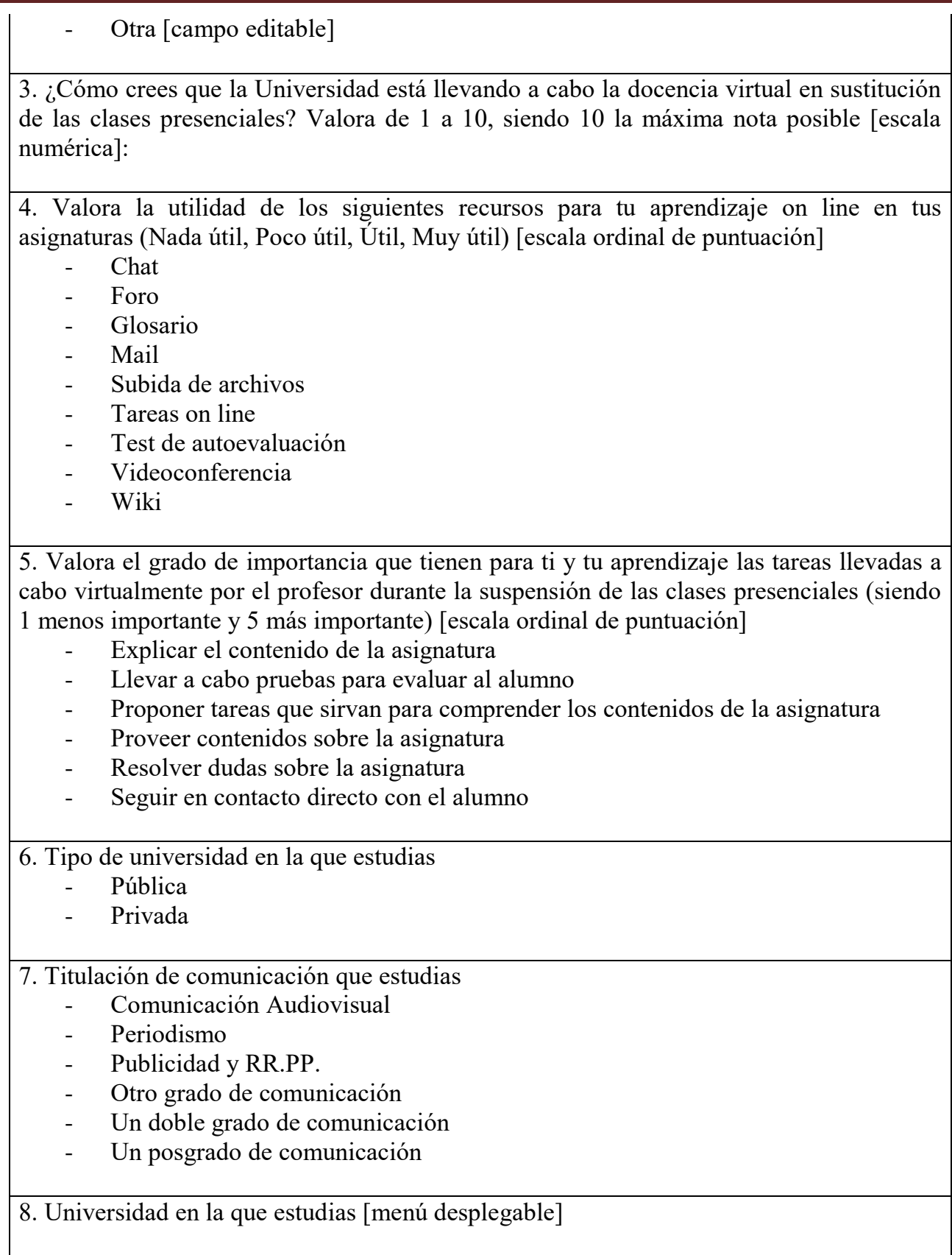

Fuente: Elaboración propia

Para enviar el cuestionario, vía mail, se creó una base de datos de contactos de profesorado de las diferentes Facultades de Comunicación españolas a quienes se les remitió para que se lo enviaran a sus alumnos; en los casos en los que se obtuvo una menor respuesta se envió el cuestionario a los representantes de alumnos de aquellos centros en los que existían organizaciones estudiantiles para que los distribuyeran entre sus compañeros. La cumplimentación por parte del alumnado fue realizada durante las semanas que duró la declaración del estado de alarma, y la consiguiente suspensión de la actividad académica presencial en todas las universidades; siempre antes del término del período lectivo que osciló entre finales de abril y finales de mayo entre las distintas universidades. Conviene apuntar que la suspensión de las clases presenciales en todo el país comenzó el 12 de marzo y se consolidó con la declaración del estado de alarma el 14 de marzo, si bien se optó por comenzar el trabajo de campo una vez pasadas dos semanas desde la declaración de dicho estado de alarma; ello fue debido a que, dado lo sobrevenido de la situación, las Facultades de 
Comunicación tuvieron que tener un margen de tiempo para poder adaptar sus dinámicas docentes presenciales a la docencia en remoto y no se quería que la valoración que los estudiantes hiciesen de ese necesario tiempo de adaptación desvirtuase los resultados finales de la investigación.

La investigación se basó en una muestra no probabilística de estudiantes de Facultades de Comunicación españolas, un total de $\mathrm{N}=560$ individuos que contestaron las preguntas de que constaba el cuestionario entre el 29 de marzo y el 5 de mayo de 2020. Como punto de partida, no se estableció como variable de estudio relevante la universidad concreta en la que realizaban sus estudios, si bien participaron estudiantes de universidades tanto públicas como privadas y de todas las Comunidades Autónomas que componen el Estado español en las cuales se ofertan estudios universitarios de comunicación (Tabla 2); ello a pesar de las dificultades que supuso, en pleno confinamiento y estado de alarma, poder contactar y contar con la colaboración de alumnos de tantas universidades. El 72,1\% de los estudiantes participantes fueron alumnos de universidades públicas y el restante $27,9 \%$ de universidades privadas. Con esta muestra se garantizaba la validez de la aproximación al objeto de estudio, establecida como objetivo principal de nuestro análisis.

Tabla 2. Comunidad Autónoma de la universidad

\begin{tabular}{|l|r|r|}
\hline \multicolumn{1}{|c|}{ Comunidad Autónoma } & \multicolumn{1}{c|}{$\begin{array}{c}\mathrm{N}^{\mathbf{}} \\
\text { Casos }\end{array}$} & $\begin{array}{c}\text { \% } \\
\text { Casos }\end{array}$ \\
\hline Comunidad de Madrid & 267 & $47,7 \%$ \\
\hline Región de Murcia & 148 & $26,4 \%$ \\
\hline Castilla y León & 60 & $10,7 \%$ \\
\hline Andalucía & 26 & $4,6 \%$ \\
\hline Aragón & 22 & $3,9 \%$ \\
\hline País Vasco & 12 & $2,1 \%$ \\
\hline Comunidad Valenciana & 6 & $1,1 \%$ \\
\hline Cataluña & 5 & $0,9 \%$ \\
\hline Galicia & 4 & $0,7 \%$ \\
\hline Canarias & 2 & $0,4 \%$ \\
\hline Cantabria & 2 & $0,4 \%$ \\
\hline Comunidad Foral de Navarra & 2 & $0,4 \%$ \\
\hline Extremadura & 2 & $0,4 \%$ \\
\hline Castilla La Mancha & 1 & $0,2 \%$ \\
\hline Islas Baleares & 1 & $0,2 \%$ \\
\hline Total & 560 & $100,0 \%$ \\
\hline
\end{tabular}

Fuente: Elaboración propia

Los datos recopilados fueron volcados en una hoja de cálculo Excel para proceder a la depuración y validación de los datos introducidos. Finalmente, se procedió al tratamiento estadístico de los datos recopilados en los cuestionarios mediante el programa IBM SPSS Statistics 24. Este software permite dos alternativas para realizar el tratamiento de la respuesta múltiple, que en el cuestionario aparece en las dos primeras preguntas, en las que el alumnado debía proporcionar información acerca de un número a priori indeterminado de ítems, a saber, los recursos docentes y las plataformas empleados en la docencia online. Se optó por el método que considera el carácter abierto de ambas preguntas (Martínez, 2006), construyendo tantas variables categóricas auxiliares como el mayor número de respuestas recogidas en cada pregunta (8 para la pregunta sobre recursos y 6 para las plataformas) por necesitar, en este caso, menos variables que el método para preguntas cerradas (que 
utilizaba variables dicotómicas en cada posible respuesta individual), y por ser asimismo más sencillo que el propuesto en Serrano Aguado, 2013. A continuación, se exponen los principales resultados hallados.

\section{Discusión y resultados}

Los resultados de la investigación indican que los estudiantes de comunicación participantes en la investigación aprueban -valga la terminología académica- la forma en que los centros en los que estudian implementaron la docencia virtual en sustitución de la presencial; de hecho, según se expone en la Tabla 3, el 57\% la puntúa por encima de 5 sobre 10; los que la califican con un notable suponen casi una cuarta parte del total $(24,5 \%)$ y los que le conceden un sobresaliente son un $8,4 \%$; por tanto, prácticamente un tercio de los estudiantes hace una valoración muy positiva. Ahora bien, no conviene obviar que el $24,1 \%$ del total de estudiantes otorga simplemente un aprobado al modo en que sus universidades desarrollaron la docencia virtual en sustitución de la presencial y que hay un porcentaje del $43 \%$ que ha optado por suspender la gestión realizada.

Por otra parte, el grado de satisfacción de los alumnos participantes en la investigación es desigual tanto entre los diferentes centros como dentro de ellos. En primer lugar, se observa claramente una mejor valoración entre los estudiantes de las universidades privadas frente a los de las públicas. Y, en segundo lugar, como se refleja en la Tabla 3, el menor grado de satisfacción se observa entre los alumnos de Comunicación Audiovisual (el 45,3\% aprueba la gestión de su universidad) frente a, por ejemplo, Periodismo $(65,2 \%)$ o Publicidad y RR.PP. (54,7\%). Los resultados indican un grado de satisfacción muy superior entre los alumnos de dobles grados o de otro tipo de grados de comunicación, si bien estos datos deben ponerse en cuestión por el número limitado de alumnos de tales estudios entre los que cumplimentaron el cuestionario.

Tabla 3. Grado de satisfacción con la docencia virtual por titulación

\begin{tabular}{|c|c|c|c|c|c|c|c|c|}
\hline Punt. & Titulación & $\begin{array}{c}\text { Comunicación } \\
\text { Audiovisual }\end{array}$ & Periodismo & $\begin{array}{c}\text { Publicidad } \\
\text { y RR.PP. }\end{array}$ & $\begin{array}{c}\text { Otro grado } \\
\text { Comunicación }\end{array}$ & $\begin{array}{c}\text { Doble grado } \\
\text { Comunicación }\end{array}$ & $\begin{array}{c}\text { Posgrado } \\
\text { Comunicación }\end{array}$ & Total \\
\hline \multirow[t]{2}{*}{1} & $\mathrm{~N}^{\mathrm{o}}$ Casos & 22 & 9 & 18 & 0 & 2 & 0 & 51 \\
\hline & $\%$ Titul. & $14,7 \%$ & $8,0 \%$ & $9,9 \%$ & $0,0 \%$ & $2,1 \%$ & $0,0 \%$ & $9,1 \%$ \\
\hline \multirow[t]{2}{*}{2} & $\mathrm{~N}^{\mathrm{o}}$ Casos & 18 & 6 & 13 & 0 & 4 & 1 & 42 \\
\hline & $\%$ Titul. & $12,0 \%$ & $5,4 \%$ & $7,2 \%$ & $0,0 \%$ & $4,3 \%$ & $20,0 \%$ & $7,5 \%$ \\
\hline \multirow[t]{2}{*}{3} & $\mathrm{~N}^{\mathrm{o}}$ Casos & 22 & 9 & 29 & 2 & 17 & 1 & 80 \\
\hline & $\%$ Titul. & $14,7 \%$ & $8,0 \%$ & $16,0 \%$ & $11,1 \%$ & $18,1 \%$ & $20,0 \%$ & $14,3 \%$ \\
\hline \multirow[t]{2}{*}{4} & $\mathrm{~N}^{\circ}$ Casos & 20 & 15 & 22 & 2 & 9 & 0 & 68 \\
\hline & $\%$ Titul. & $13,3 \%$ & $13,4 \%$ & $12,2 \%$ & $11,1 \%$ & $9,6 \%$ & $0,0 \%$ & $12,1 \%$ \\
\hline \multirow[t]{2}{*}{5} & $\mathrm{~N}^{\mathrm{o}}$ Casos & 15 & 11 & 19 & 1 & 8 & 1 & 55 \\
\hline & $\%$ Titul. & $10,0 \%$ & $9,8 \%$ & $10,5 \%$ & $5,6 \%$ & $8,5 \%$ & $20,0 \%$ & $9,8 \%$ \\
\hline \multirow[t]{2}{*}{6} & $\mathrm{~N}^{\circ}$ Casos & 14 & 18 & 26 & 1 & 20 & 1 & 80 \\
\hline & $\%$ Titul. & $9,3 \%$ & $16,1 \%$ & $14,4 \%$ & $5,6 \%$ & $21,3 \%$ & $20,0 \%$ & $14,3 \%$ \\
\hline \multirow[t]{2}{*}{7} & $\mathrm{~N}^{\mathrm{o}}$ Casos & 18 & 23 & 19 & 8 & 23 & 0 & 91 \\
\hline & $\%$ Titul. & $12,0 \%$ & $20,5 \%$ & $10,5 \%$ & $44,4 \%$ & $24,5 \%$ & $0,0 \%$ & $16,3 \%$ \\
\hline \multirow[t]{2}{*}{8} & $\mathrm{~N}^{\circ}$ Casos & 6 & 11 & 20 & 2 & 6 & 1 & 46 \\
\hline & $\%$ Titul. & $4,0 \%$ & $9,8 \%$ & $11,0 \%$ & $11,1 \%$ & $6,4 \%$ & $20,0 \%$ & $8,2 \%$ \\
\hline 9 & $\mathrm{~N}^{\mathrm{o}} \mathrm{Casos}$ & 7 & 3 & 7 & 2 & 2 & 0 & 21 \\
\hline
\end{tabular}


RLCS, Revista Latina de Comunicación Social, 78, 265-287

[Investigación] DOI: 10.4185/RLCS-2020-1477| ISSN 1138-5820| Año 2020

\begin{tabular}{|c|l|r|r|r|r|r|r|r|}
\hline \hline & $\%$ Titul. & $4,7 \%$ & $2,7 \%$ & $3,9 \%$ & $11,1 \%$ & $2,1 \%$ & $0,0 \%$ & $3,8 \%$ \\
\hline 10 & $\mathrm{~N}^{\circ}$ Casos & 8 & 7 & 8 & 0 & 3 & 0 & 26 \\
\hline & $\%$ Titul. & $5,3 \%$ & $6,3 \%$ & $4,4 \%$ & $0,0 \%$ & $3,2 \%$ & $0,0 \%$ & $4,6 \%$ \\
\hline Total & $\mathrm{N}^{\circ}$ Casos & 150 & 112 & 181 & 18 & 94 & 5 & 560 \\
\hline & $\%$ Titul. & $100,0 \%$ & $100,0 \%$ & $100,0 \%$ & $100,0 \%$ & $100,0 \%$ & $100,0 \%$ & $100,0 \%$ \\
\hline
\end{tabular}

Fuente: Elaboración propia

Además de esa valoración global, con la investigación realizada se persiguió conocer cuáles eran los recursos docentes y las plataformas tecnológicas que se utilizaron durante la docencia en remoto. Comenzando por las plataformas (Tabla 4), se obtuvieron un total de 1.117 respuestas ya que los encuestados podían señalar varias posibilidades hasta un máximo de 3 , y se observa claramente que la principal plataforma es el aula virtual de la universidad. Analizando los datos en función del número de encuestados, cabe señalar que el $84.5 \%$ afirma haber usado el aula virtual y el 32,5\% Blackboard Collaborate, plataformas cuyos datos podrían unificarse por estar esta última herramienta frecuentemente integrada en el aula virtual. Le sigue Microsoft Teams $(28,0 \%)$ o redes sociales $(12,7 \%)$; entre una y otra, aproximadamente 1 de cada 10 estudiantes también afirma haber usado otras plataformas como blogs o sites de la asignatura y Zoom. Es muy inferior el uso de otras plataformas como Skype, Whastapp, WebEx, Google Meet o Hangouts.

Tabla 4. Plataformas tecnológicas utilizadas

\begin{tabular}{|l|r|r|r|}
\hline \multicolumn{1}{|c|}{ Plataformas } & \multicolumn{1}{c|}{$\begin{array}{c}\mathrm{N}^{\circ} \\
\text { Resp. }\end{array}$} & $\begin{array}{c}\text { Resp. } \\
\text { Res }\end{array}$ & $\begin{array}{c}\text { Casos } \\
\text { Cula virtual de universidad }\end{array}$ \\
\hline Blackboard Collaborate & 182 & $42,3 \%$ & $84,5 \%$ \\
\hline Microsoft Teams & 157 & $14,1 \%$ & $28,0 \%$ \\
\hline Redes sociales & 71 & $6,4 \%$ & $12,7 \%$ \\
\hline Blogs y espacios web asignatura & 64 & $5,7 \%$ & $11,4 \%$ \\
\hline Zoom & 64 & $5,7 \%$ & $11,4 \%$ \\
\hline Skype & 40 & $3,6 \%$ & $7,1 \%$ \\
\hline Whatsapp & 29 & $2,6 \%$ & $5,2 \%$ \\
\hline WebEx & 16 & $1,4 \%$ & $2,9 \%$ \\
\hline Google Meet & 13 & $1,2 \%$ & $2,3 \%$ \\
\hline Ninguno & 4 & $0,4 \%$ & $0,7 \%$ \\
\hline Hangouts & 4 & $0,4 \%$ & $0,7 \%$ \\
\hline Total & 1.117 & $100,0 \%$ & \\
\hline
\end{tabular}

Fuente: Elaboración propia

En todo caso, lo más habitual ha sido la utilización de varias plataformas simultáneamente. Los resultados de nuestra investigación (Tabla 5) muestran que la mayoría de los estudiantes encuestados utilizaron varias plataformas tecnológicas (el 30,0\%, con tres; el 28,4\%, con dos), si bien hay un 37,5\% del total que manifiesta haber usado sólo una; mientras tanto, sólo un 3,4\% de los estudiantes señala haber trabajado en sus cursos de comunicación con más de tres plataformas distintas. Por estudios de grado, es en la titulación de Publicidad y RR.PP. donde se ha utilizado un mayor número de plataformas distintas; por ejemplo, el 37,5\% de los estudiantes que manifiesta haber utilizado una sola plataforma baja al 32,0\% en el caso de Publicidad y RR.PP. mientras que sube al 52,7\% y 47,3\% en el caso de Periodismo y Comunicación Audiovisual, respectivamente.

Tabla 5. Número de plataformas utilizadas por titulación 
RLCS, Revista Latina de Comunicación Social, 78, 265-287

[Investigación] DOI: 10.4185/RLCS-2020-1477| ISSN 1138-5820| Año 2020

\begin{tabular}{|c|c|c|c|c|c|c|c|c|}
\hline $\begin{array}{l}\mathrm{N}^{\circ} \text {. de } \\
\text { plataf. }\end{array}$ & Titulación & $\begin{array}{c}\text { Comunicación } \\
\text { Audiovisual } \\
\end{array}$ & Periodismo & \begin{tabular}{|c|} 
Publicidad \\
y RR.PP. \\
\end{tabular} & $\begin{array}{c}\text { Otro grado } \\
\text { comunicación }\end{array}$ & $\begin{array}{l}\text { Doble grado } \\
\text { comunicación }\end{array}$ & $\begin{array}{c}\text { Posgrado } \\
\text { comunicación }\end{array}$ & Total \\
\hline \multirow[t]{2}{*}{0} & Recuento & 1 & 1 & 2 & 0 & 0 & 0 & 4 \\
\hline & $\%$ Titul. & $0,7 \%$ & $0,9 \%$ & $1,1 \%$ & $0,0 \%$ & $0,0 \%$ & $0,0 \%$ & $0,7 \%$ \\
\hline \multirow[t]{2}{*}{1} & Recuento & 71 & 59 & 58 & 2 & 16 & 4 & 210 \\
\hline & $\%$ Titul. & $47,3 \%$ & $52,7 \%$ & $32,0 \%$ & $11,1 \%$ & $17,0 \%$ & $80,0 \%$ & $37,5 \%$ \\
\hline \multirow[t]{2}{*}{2} & Recuento & 62 & 23 & 41 & 7 & 25 & 1 & 159 \\
\hline & $\%$ Titul. & $41,3 \%$ & $20,5 \%$ & $22,7 \%$ & $38,9 \%$ & $26,6 \%$ & $20,0 \%$ & $28,4 \%$ \\
\hline \multirow[t]{2}{*}{3} & Recuento & 15 & 28 & 68 & 8 & 49 & 0 & 168 \\
\hline & $\%$ Titul. & $10,0 \%$ & $25,0 \%$ & $37,6 \%$ & $44,4 \%$ & $52,1 \%$ & $0,0 \%$ & $30,0 \%$ \\
\hline \multirow[t]{2}{*}{4} & Recuento & 0 & 0 & 10 & 1 & 2 & 0 & 13 \\
\hline & $\%$ Titul. & $0,0 \%$ & $0,0 \%$ & $5,5 \%$ & $5,6 \%$ & $2,1 \%$ & $0,0 \%$ & $2,3 \%$ \\
\hline \multirow[t]{2}{*}{5} & Recuento & 1 & 0 & 2 & 0 & 2 & 0 & 5 \\
\hline & $\%$ Titul. & $0,7 \%$ & $0,0 \%$ & $1,1 \%$ & $0,0 \%$ & $2,1 \%$ & $0,0 \%$ & $0,9 \%$ \\
\hline \multirow[t]{2}{*}{6} & $\mathrm{~N}^{\mathrm{o}}$ Casos & 0 & 1 & 0 & 0 & 0 & 0 & 1 \\
\hline & $\%$ Titul. & $0,0 \%$ & $0,9 \%$ & $0,0 \%$ & $0,0 \%$ & $0,0 \%$ & $0,0 \%$ & $0,2 \%$ \\
\hline \multirow[t]{2}{*}{ Total } & $\mathrm{N}^{\mathrm{o}}$ Casos & 150 & 112 & 181 & 18 & 94 & 5 & 560 \\
\hline & $\%$ Titul. & $100,0 \%$ & $100,0 \%$ & $100,0 \%$ & $100,0 \%$ & $100,0 \%$ & $100,0 \%$ & $100,0 \%$ \\
\hline
\end{tabular}

Fuente: Elaboración propia

Respecto a los recursos docentes empleados en la docencia virtual de las asignaturas, lo más frecuente ha sido la utilización de tres recursos distintos (así lo manifiesta el 58\% de los encuestados) y a continuación dos $(13,4 \%)$; sólo un $6,8 \%$ de los estudiantes encuestados declara haber usado tan sólo un recurso y existe un 1,1\% marginal que afirma no haber utilizado ninguno, lo cual implica la inexistencia de docencia virtual de ningún tipo. El restante $20,7 \%$ corresponde a alumnos que disfrutaron en sus asignaturas de más de tres recursos. Los porcentajes son similares tanto entre universidades públicas como privadas, aunque se observa una mayor tendencia a la diversificación de los recursos en éstas últimas.

Tabla 6. Número de recursos docentes utilizados por tipo de universidad

\begin{tabular}{|c|l|r|r|r|}
\hline $\begin{array}{c}\mathrm{N}^{\mathrm{o}} . \text { de } \\
\text { recursos }\end{array}$ & $\begin{array}{c}\text { Tipo de } \\
\text { universidad }\end{array}$ & Pública & Privada & \multicolumn{1}{c|}{ Total } \\
\hline 0 & $\mathrm{~N}^{\mathrm{o}}$ Casos & 6 & 0 & 6 \\
\hline & $\%$ Tipo & $1,5 \%$ & $0,0 \%$ & $1,1 \%$ \\
\hline 1 & $\mathrm{~N}^{\mathrm{o}}$ Casos & 24 & 14 & 38 \\
\hline & $\%$ Tipo & $5,9 \%$ & $9,0 \%$ & $6,8 \%$ \\
\hline 2 & $\mathrm{~N}^{\mathrm{o}}$ Casos & 69 & 6 & 75 \\
\hline & $\%$ Tipo & $17,1 \%$ & $3,8 \%$ & $13,4 \%$ \\
\hline 3 & $\mathrm{~N}^{\mathrm{o}}$ Casos & 230 & 95 & 325 \\
\hline & $\%$ Tipo & $56,9 \%$ & $60,9 \%$ & $58,0 \%$ \\
\hline 4 & $\mathrm{~N}^{\mathrm{o}}$ Casos & 37 & 11 & 48 \\
\hline & $\%$ Tipo & $9,2 \%$ & $7,1 \%$ & $8,6 \%$ \\
\hline 5 & $\mathrm{~N}^{\circ}$ Casos & 26 & 19 & 45 \\
\hline & $\%$ Tipo & $6,4 \%$ & $12,2 \%$ & $8,0 \%$ \\
\hline 6 & $\mathrm{~N}^{\circ}$ Casos & 10 & 10 & 20 \\
\hline & $\%$ Tipo & $2,5 \%$ & $6,4 \%$ & $3,6 \%$ \\
\hline
\end{tabular}


RLCS, Revista Latina de Comunicación Social, 78, 265-287

[Investigación] DOI: 10.4185/RLCS-2020-1477| ISSN 1138-5820| Año 2020

\begin{tabular}{|c|l|r|r|r|}
\hline 7 & $\mathrm{~N}^{\circ}$ Casos & 2 & 0 & 2 \\
\hline & $\%$ Tipo & $0,5 \%$ & $0,0 \%$ & $0,4 \%$ \\
\hline 8 & $\mathrm{~N}^{\circ}$ Casos & 0 & 1 & 1 \\
\hline & $\%$ Tipo & $0,0 \%$ & $0,6 \%$ & $0,2 \%$ \\
\hline Total & $\mathrm{N}^{\circ}$ Casos & 404 & 156 & 560 \\
\hline & $\%$ Tipo & $100,0 \%$ & $100,0 \%$ & $100,0 \%$ \\
\hline
\end{tabular}

Fuente: Elaboración propia

Por otra parte, no se pueden concluir diferencias muy importantes entre las respuestas de los estudiantes encuestados de las distintas titulaciones de comunicación. Ahora bien, centrándonos en los tres grados clásicos de Periodismo, Comunicación Audiovisual y Publicidad y RR.PP., se observa que en el caso de Periodismo se da menor diversidad en los recursos docentes usados por los profesores; no en vano si sobre el total de la muestra el 79,3\% de los estudiantes tuvieron a su disposición menos de tres recursos, en el caso de Periodismo sube hasta el 86,6\%, seguido de Comunicación Audiovisual (80,0\%), siendo el grado de Publicidad y RR.PP. aquél en que los docentes ofrecieron un mayor número de recursos, según los encuestados.

Concretando cuáles han sido los principales recursos docentes utilizados por los profesores, de las 1.725 respuestas obtenidas se extraen los siguientes datos. El 73,2\% de los estudiantes que participaron en la investigación señala que sus profesores han subido archivos con contenidos del temario de la asignatura, el $67,9 \%$ que han realizado videoconferencias y el 58,9\% que han propuesto tareas online ligadas a los aspectos prácticos de las asignaturas. Estos han sido los principales recursos utilizados por los docentes de comunicación, además de otros como el mail $(42,3 \%)$, los foros $(34,1 \%)$ o los chats $(23,6 \%)$, más orientados a la participación y retroalimentación con el alumnado. En la Tabla 7 se pueden observar los porcentajes específicos totales y desagregados por tipo de universidad. En ambos tipos de universidades, los recursos más mencionados fueron la subida de archivos, la videoconferencia y las tareas online, con más del 60\% de las respuestas en cada caso, aunque en diferente orden (siendo la más importante la subida de archivos en la universidad pública vs. la videoconferencia en la privada); y sólo en el caso de la universidad pública se recogió como respuesta la ausencia de recurso alguno. Un test de homogeneidad chi-cuadrado reveló diferencias significativas en los recursos usados entre ambos tipos de universidades (valor del test $=165,524$ con $\mathrm{p}=0,000$ ) luego puede afirmarse que los recursos son utilizados de manera diferente en los dos casos.

Tabla 7. Recursos docentes utilizados por tipo de universidad

\begin{tabular}{|c|c|c|c|c|c|c|}
\hline \multirow[b]{2}{*}{ Recursos utilizados } & \multicolumn{2}{|c|}{ Pública } & \multicolumn{2}{|c|}{ Privada } & \multicolumn{2}{|c|}{ Total } \\
\hline & $\begin{array}{c}\mathrm{N}^{\mathrm{o}} \\
\text { Resp. }\end{array}$ & $\%$ & $\begin{array}{c}\mathrm{N}^{\mathrm{o}} \\
\text { Resp. }\end{array}$ & $\%$ & $\begin{array}{c}\mathrm{N}^{\mathrm{o}} \\
\text { Resp. }\end{array}$ & $\%$ \\
\hline Subida de archivos (temario) & 313 & $25,9 \%$ & 97 & $18,7 \%$ & 410 & $23,8 \%$ \\
\hline Videoconferencia & 233 & $19,3 \%$ & 147 & $28,4 \%$ & 380 & $22,0 \%$ \\
\hline Tareas online (prácticas) & 223 & $18,5 \%$ & 107 & $20,7 \%$ & 330 & $19,1 \%$ \\
\hline Mail & 161 & $13,3 \%$ & 76 & $14,7 \%$ & 237 & $13,7 \%$ \\
\hline Foro & 172 & $14,3 \%$ & 19 & $3,7 \%$ & 191 & $11,1 \%$ \\
\hline Chat & 78 & $6,5 \%$ & 54 & $10,4 \%$ & 132 & $7,7 \%$ \\
\hline Test de autoevaluación & 17 & $1,4 \%$ & 16 & $3,1 \%$ & 33 & $1,9 \%$ \\
\hline Ninguno & 6 & $0,5 \%$ & 0 & $0,0 \%$ & 6 & $0,3 \%$ \\
\hline Glosario & 3 & $0,2 \%$ & 1 & $0,2 \%$ & 4 & $0,2 \%$ \\
\hline Wiki & 1 & $0,1 \%$ & 1 & $0,2 \%$ & 2 & $0,1 \%$ \\
\hline
\end{tabular}


RLCS, Revista Latina de Comunicación Social, 78, 265-287

[Investigación] DOI: 10.4185/RLCS-2020-1477| ISSN 1138-5820| Año 2020

\begin{tabular}{|l|l|l|l|l|l|l|} 
Total & 1.207 & $100,0 \%$ & 518 & $100,0 \%$ & 1.725 & $100,0 \%$ \\
\hline
\end{tabular}

Fuente: Elaboración propia

Además de identificar cuáles eran los principales recursos docentes utilizados en la docencia en remoto por los profesores de la Facultades de Comunicación, otro objetivo de la investigación realizada era conocer la valoración que los estudiantes participantes en la investigación hacen de dichos recursos. A partir de la Tabla 8 se puede observar qué grado de utilidad ven los alumnos en aquellos recursos que previamente han respondido que han sido empleados. Se comprobó, por otra parte, que la valoración que los alumnos hacían de los recursos que habían utilizado diferían respecto de aquellos casos en los que opinaban sin haberlos utilizado. Pues bien, se podría decir que los recursos más usados son también, a grandes rasgos, los que los alumnos consideran más útiles. No en vano, el principal es la subida de archivos, utilizada por 410 alumnos, que ellos consideran en un 78,1\% útil o muy útil, seguido de la videoconferencia con 380 alumnos (útil o muy útil para el 82,6\%), las tareas online (para el 69,1\%) y el mail (para el 74,7\%). Mientras tanto, la percepción de utilidad es menor en el caso de recursos menos utilizados como los foros o los chats, que los alumnos solo consideran útiles o muy útiles en el $47,6 \%$ y 55,3\% de los casos respectivamente, y todavía inferior en otros recursos como glosarios o wikis. Ahora bien, también cabría destacar dos datos significativos. De un lado, la alta valoración de utilidad que los alumnos hacen de los test de autoevaluación a pesar de ser un recurso poco utilizado por los profesores (el 75,7\% de los alumnos que los usaron los consideran útiles o muy útiles pesa a ser un recurso que sólo el 5,9\% de los estudiantes afirma que sus profesores les han ofrecido); en todo caso es un dato que debieran ser contrastado con una muestra más amplia. Y de otro lado, la alta utilidad que los alumnos otorgan a la videoconferencia como recurso docente, dado que es el que los estudiantes califican como muy útil en el mayor porcentaje de casos, aunque no haya sido el recurso más utilizado por los profesores.

Tabla 8. Utilidad percibida de los recursos docentes utilizados

\begin{tabular}{|c|c|c|c|c|c|c|c|c|c|c|}
\hline \multirow{2}{*}{$\begin{array}{l}\text { Recursos } \\
\text { utilizados }\end{array}$} & \multicolumn{2}{|c|}{ Nada útil } & \multicolumn{2}{|c|}{ Poco útil } & \multicolumn{2}{|c|}{ Útil } & \multicolumn{2}{|c|}{ Muy útil } & \multicolumn{2}{|c|}{ Total } \\
\hline & $\begin{array}{c}\mathrm{N}^{\mathrm{o}} \\
\text { Resp. }\end{array}$ & $\%$ & $\begin{array}{c}\mathrm{N}^{\circ} \\
\text { Resp. }\end{array}$ & $\%$ & $\begin{array}{c}\mathrm{N}^{\circ} \\
\text { Resp. }\end{array}$ & $\%$ & $\begin{array}{c}\mathrm{N}^{\mathrm{o}} \\
\text { Resp. }\end{array}$ & $\%$ & $\begin{array}{c}\mathrm{N}^{\circ} \\
\text { Resp. }\end{array}$ & $\%$ \\
\hline $\begin{array}{ll}\text { Subida } & \text { de } \\
\text { archivos } & \\
\text { (temario) } & \\
\end{array}$ & 20 & $4,9 \%$ & 70 & $17,1 \%$ & 170 & $41,5 \%$ & 150 & $36,6 \%$ & 410 & $100,0 \%$ \\
\hline Videoconferencia & 14 & $3,7 \%$ & 52 & $13,7 \%$ & 149 & $39,2 \%$ & 165 & $43,4 \%$ & 380 & $100,0 \%$ \\
\hline $\begin{array}{l}\text { Tareas online } \\
\text { (prácticas) }\end{array}$ & 27 & $8,2 \%$ & 75 & $22,7 \%$ & 149 & $45,2 \%$ & 79 & $23,9 \%$ & 330 & $100,0 \%$ \\
\hline Mail & 20 & $8,4 \%$ & 40 & $16,9 \%$ & 105 & $44,3 \%$ & 72 & $30,4 \%$ & 237 & $100,0 \%$ \\
\hline Foro & 16 & $8,4 \%$ & 84 & $44,0 \%$ & 69 & $36,1 \%$ & 22 & $11,5 \%$ & 191 & $100,0 \%$ \\
\hline Chat & 15 & $11,4 \%$ & 44 & $33,3 \%$ & 50 & $37,9 \%$ & 23 & $17,4 \%$ & 132 & $100,0 \%$ \\
\hline $\begin{array}{l}\text { Test de } \\
\text { autoevaluación }\end{array}$ & 1 & $3,0 \%$ & 7 & $21,2 \%$ & 14 & $42,4 \%$ & 11 & $33,3 \%$ & 33 & $100,0 \%$ \\
\hline Glosario & 0 & $0,0 \%$ & 3 & $75,0 \%$ & 0 & $0,0 \%$ & 1 & $25,0 \%$ & 4 & $100,0 \%$ \\
\hline Wiki & 0 & $0,0 \%$ & 1 & $50,0 \%$ & 1 & $50,0 \%$ & 0 & $0,0 \%$ & 2 & $100,0 \%$ \\
\hline Total & --- & --- & --- & --- & --- & --- & --- & --- & 560 & $100,0 \%$ \\
\hline
\end{tabular}

Fuente: Elaboración propia

Por otra parte, con independencia de los recursos y plataformas utilizadas, también se preguntó a los estudiantes por el grado de importancia que tienen para ellos las diferentes tareas que los profesores 
pudieron llevar a cabo virtualmente durante la suspensión de las clases presenciales; se propuso una valoración de esa importancia mediante una escala de Likert de 1 a 5 . Lo más importante para el alumnado (Tabla 9) resultó, por un lado, que el profesor resuelva dudas sobre la asignatura y explique el contenido de la misma, es decir, dos misiones esenciales en la labor docente; la primera la puntúan con 5 en el 55,2\% de los casos, y con 4 y 5 en el 79,7\%, mientras que la segunda tiene un grado de importancia de 5 también para más de la mitad de los encuestados $(51,8 \%$ ) y la valoración entre 4 y 5 alcanza casi los tres cuartos $(73,9 \%)$. Por otro lado, junto a ellas otra tarea importante para los estudiantes es la de seguir en contacto directo con el profesor, ya que tiene la máxima importancia para el 51,8\% del alumnado que la puntúa con 5 y un 77,2\% le da una puntuación de 4 ó 5. Proveer contenidos sobre la asignatura es la siguiente tarea más importante para el alumnado, dado que el $66,4 \%$ la puntúa entre 4 y 5 . Por el contrario, lo menos valorado en términos de importancia para los estudiantes, son las tareas prácticas y, sobre todo, las pruebas de evaluación encargadas a los estudiantes: proponer tareas que sirvan para comprender los contenidos es valorado con 4 y 5 por el $57,7 \%$ de los estudiantes, mientras que llevar a cabo virtualmente pruebas para evaluar el aprendizaje del alumnado obtiene en el 63\% de los casos una puntuación igual o inferior a 3.

Tabla 9. Importancia de las tareas docentes online

\begin{tabular}{|c|r|r|r|r|r|r|}
\hline Punt. & Explicaciones & Pruebas & \multicolumn{1}{c|}{ Tareas } & \multicolumn{1}{c|}{ Contenidos } & \multicolumn{1}{c|}{ Dudas } & \multicolumn{1}{c|}{ Contacto } \\
\hline 1 & $5,0 \%$ & $10,5 \%$ & $5,7 \%$ & $3,9 \%$ & $2,9 \%$ & $4,3 \%$ \\
\hline 2 & $5,0 \%$ & $18,6 \%$ & $12,9 \%$ & $8,6 \%$ & $4,5 \%$ & $5,4 \%$ \\
\hline 3 & $16,1 \%$ & $33,9 \%$ & $23,8 \%$ & $21,1 \%$ & $13,0 \%$ & $13,2 \%$ \\
\hline 4 & $22,1 \%$ & $24,1 \%$ & $30,4 \%$ & $28,9 \%$ & $24,5 \%$ & $25,4 \%$ \\
\hline 5 & $51,8 \%$ & $12,9 \%$ & $27,3 \%$ & $37,5 \%$ & $55,2 \%$ & $51,8 \%$ \\
\hline Total & $100,0 \%$ & $100,0 \%$ & $100,0 \%$ & $100,0 \%$ & $100,0 \%$ & $100,0 \%$ \\
\hline
\end{tabular}

Fuente: Elaboración propia

\section{Conclusiones}

La crisis sanitaria de la COVID-19 ha forzado a las universidades españolas -y en consecuencia a las Facultades de Comunicación- a afrontar el reto de su transformación digital, en particular la adaptación de la docencia presencial a entornos virtuales. Si bien informes previos sobre el uso de las TIC en el contexto universitario español arrojaban resultados positivos, lo cierto es que la pandemia ha evidenciado las debilidades en la infraestructura tecnológica de las universidades o en la formación del profesorado para dar respuesta a planes de blended-learning o incluso a la efectiva implantación total de la docencia en remoto. En la investigación desarrollada se ha querido saber cómo los estudiantes de comunicación han percibido la adaptación de sus centros de estudio a esa docencia virtual y los resultados revelan opiniones diversas. No en vano, aunque la valoración positiva es superior a la negativa, cabría hacer algunos matices. En primer lugar, el mayor grado de satisfacción de los estudiantes encuestados que estudian en universidades privadas; sin poder valorar los porqués, de las opiniones de los estudiantes se deduce claramente que estos centros parecen haberse mostrado mucho más flexibles y resolutivos para la gestión de la situación y la rápida implementación de su total docencia online. En segundo lugar, el menor grado de satisfacción en aquellas titulaciones con un mayor componente práctico o de docencia en laboratorio, como por ejemplo Comunicación Audiovisual, es decir la que tradicionalmente no se ha impartido online por su especificidad. 
En general, se podría decir que las Facultades de Comunicación -en el contexto de la universidad española- han sabido potenciar los recursos de los que disponían para la docencia virtual; así, que las universidades convirtiesen el aula virtual, y sus aplicaciones asociadas, en la plataforma tecnológica de referencia para la docencia ha evitado una mayor imagen de descoordinación como la vivida en otros niveles educativos. En contrapartida, de los resultados de nuestra investigación también se deduce que a grandes rasgos se han trasladado al mundo online los métodos docentes propios de la enseñanza presencial en lugar de desarrollar una docencia que aproveche todas las potencialidades de lo virtual; muestra de ello es que el principal recurso docente utilizado por los profesores haya sido la subida de archivos con contenidos teóricos de las asignaturas o que la videoconferencia, recurso mejor valorado por los estudiantes, no fuera tan utilizado por los docentes de las distintas universidades. Es entendible, en todo caso, teniendo en cuenta que la adaptación tuvo que hacerse a marchas forzadas y con soportes tecnológicos limitados.

Por último, conviene destacar que las opiniones de los estudiantes han sido recogidas en un contexto de gran excepcionalidad que probablemente haya influido en la comprensión de parte del alumnado hacia la gestión de sus centros, por lo que sería necesario seguir investigando en qué medida la docencia virtual ha servido o no para alcanzar los objetivos de aprendizaje de las distintas titulaciones de comunicación. El hecho de que los encuestados reconozcan que en la labor del profesor durante la docencia online es prácticamente tan importante mantener el contacto con los alumnos como otras cuestiones más vinculadas a la explicación de contenidos o resolución de dudas demuestra lo fundamental que es en la misión docente no sólo la transmisión de conocimientos sino también la atención al alumno, más aún en una situación social anómala como la analizada en esta investigación. Es necesario, en todo caso, llevar a cabo nuevos estudios que complemente la aproximación aquí presentada, sirvan para contrastar los resultados obtenidos y permiten seguir conociendo la evolución de la labor docente virtual llevada a cabo en los estudios universitarios de comunicación en España. Dichos estudios debieran superar las limitaciones del aquí presentado, dado que el proceso de recogida de datos se tuvo que hacer en unas condiciones muy complejas debido a la declaración del estado de alarma; especialmente en lo que se refiere a una necesaria mayor equidad en la muestra con la que se ha trabajado, si bien en todo caso la investigación realizada se ha presentado como una aproximación descriptiva al fenómeno analizado. Puesto que durante el curso académico 2020-2021 la actividad académica en las universidades españolas seguirá condicionada por la situación sanitaria provocada por la COVID-19 es conveniente realizar investigaciones que amplíen y completen la actual, profundizando aún más en el conocimiento de las dinámicas de adaptación de la docencia a los entornos virtuales.

\section{Bibliografía}

Aparici, R. \& García-Matilla, A. (2016) ¿Qué ha ocurrido con la educación en comunicación en los últimos 35 años?: pensar el futuro. Espacios en Blanco. Revista de Educación (Serie Indagaciones), $\quad 26, \quad$ pp. 35-57. https://digital.cic.gba.gob.ar/bitstream/handle/11746/6184/11746_6184.pdfPDFA.pdf? sequence $=1 \&$ isAllowed $=y$

Baelo Álvarez, R. \& Cantón Mayo, I. (2009). Las tecnologías de la información y la comunicación en la educación superior. Estudio descriptivo y de revisión. Revista Iberoamericana de Educación, 50 (7), pp. 1-12. https://doi.org/10.35362/rie5071965

Barro Ameneiro, S. (dir.) (2004). Las Tecnologías de la Información y la Comunicación en el Sistema Universitario Español. CRUE. 
Bartolomé-Pina, A.; García-Ruiz, R. \& Aguaded, I. (2018). Blended learning: panorama y perspectivas. RIED. Revista Iberoamericana de Educación a Distancia, 21(1), pp. 33-56.

http://dx.doi.org/10.5944/ried.21.1.18842

Berná Sicilia, C.; De Diego González, A.; Rodríguez Ruibal, A. \& Rubio Jordán, A.V. (2010). El grado de Periodismo en la Universidad a Distancia de Madrid. En J. Sierra (coord.) Los estudios de Ciencias de la Comunicación en el EESS (pp. 278-292). Fragua.

Boyd, D. (2002). Faceted id/entity: Managing representation in a digital world. Massachusetts Institute of Technology.

Bricall, J.M. (2000). Universidad 2000. CRUE. https://www.oei.es/historico/oeivirt/bricall.htm

Cabero-Almenara, J. (2020). Aprendiendo del tiempo de la COVID-19. Revista Electrónica Educare 24, Suplemento Especial, pp. 1-3

Castañeda, L. \& Selwyn, N. (eds.) (2020). Reiniciando la universidad. Buscando un modelo de universidad en tiempos digitales. Editorial UOC.

Castells, M. (2001). Internet y la sociedad red. Letra internacional, 71, pp. 4-16.

Castells, M. (2006). La sociedad red: una visión global. Alianza.

Chiappe, A. (2016). Tendencias sobre contenidos educativos digitales en América Latina. (Cuaderno SITEAL). Buenos Aires, Argentina: UNESCO. http://unesdoc.unesco.org/images/0024/002456/245673s.pdf

Coaten, N. (2003). Blended e-learning. Educaweb, https://www.educaweb.com/esp/servicios/monografico/formacionvirtual/1181076-a.html

69.

Colomer, J.; Serra, T.; Cañabate, D.; \& Bubnys, R. (2020). Reflective learning in higher education: Active methodologies for transformative practices. Sustainability, 12 (9), pp. 1-8. https://doi.org/10.3390/su12093827

Coll, C. \& Marti, E (2001). La educación escolar ante las nuevas tecnologías de la información y la comunicación. En J. Palacios et al. (Eds.), Desarrollo psicológico y educación. Vol. 2, (Psicología de la educación escolar). Alianza.

Consejo de Universidades (2003). Plan Nacional de Evaluación de la Calidad de las Universidades. Informe Global (1996-2000). Consejo de Universidades.

CRUE (2017). UNIVERSITEC 2017: análisis de las TIC en las Universidades Españolas. CRUE.

CRUE (2020a). Comunicado por la declaración del estado de alarma a consecuencia de la pandemia del

Covid-19.

http://www.crue.org/Documentos\%20compartidos/Comunicados/2020.03.14-

Comunicado\%20estado\%20alarma.pdf 
RLCS, Revista Latina de Comunicación Social, 78, 265-287

[Investigación] DOI: 10.4185/RLCS-2020-1477| ISSN 1138-5820| Año 2020

CRUE (2020b) Crue y los Ministerios de Asuntos Exteriores y de Universidades se coordinan para organizar el regreso de los estudiantes y personal universitario. http://www.crue.org/Comunicacion/Noticias/Crue\%20y\%20ministerios\%20de\%20universidades $\% 20 \mathrm{y} \% 20$ Exteriores $\% 20$ facilitan $\% 20$ regreso\%20de\%20erasmus.aspx

Cubeles, A. \& Riu. D. (2018). The effective integration of ICTs in universities: the role of knowledge and academic experience of professors. Technology, Pedagogy and Education, 27 (3), pp. 339-349. https://doi.org/10.1080/1475939X.2018.1457978

De Casas, P.; Caldeiro, M. C. \& Havrankova, T. (2020). The Knowledge and the Use of Educational Applications from University Students in Spain and the Czech Republic. Research in Education \& Learning Innovation Archives (REALIA), 24, pp. 39-55. https://doi.org/10.7203/realia.24.16696

De Pablos, J.M.; Colás, M.P.; López, A. \& García, I. (2019). Uses of digital platforms in Higher Education from the perspectives of the educational research. REDU. Revista de Docencia Universitaria, 17(1), pp. 59-72. https://doi.org/10.4995/redu.2019.11177

Decreto 2070/1971, de 13 de agosto, por el que se regulan los estudios de periodismo y demás medios de comunicación social en la universidad. https://www.boe.es/boe/dias/1971/09/14/pdfs/A14944-14945.pdf

Espinar, L. (2020, 9 de abril). La UCLM pulsa también al profesorado y los recursos tecnológicos del alumnado en otras dos consultas. Lanza Digital.com. https://www.lanzadigital.com/castilla-lamancha/la-uclm-pulsara-tambien-al-profesorado-y-los-recursos-tecnologicos-del-alumnado-enotras-dos-encuestas/

Esteves, M.; Pereira, A.; Veiga, N.; Vasco, R. \& Veiga, A. (2018). The use of new learning technologies in higher education classroom. International Journal of Engineering Pedagogy (iJEP), 8 (2), pp. 115-127. https://doi.org/10.3991/ijep.v8i2.8146

Frente de Estudiantes (2020). El sistema educativo en la crisis del COVID19. Análisis de la situación $y$ propuestas del y para el estudiantado, https://www.frentedeestudiantes.es/wpcontent/uploads/2020/04/Informe_COVID19.pdf

Fundación CYD (2020, 7 de mayo). "La pandemia nos arrebató todo”: 8 estudiantes detallan el efecto del coronavirus. https://www.fundacioncyd.org/estudiantes-impacto-del-coronavirus-en-laensenanzal

García-Aretio, L. (2004). Viejos y nuevos modelos de educación a distancia. Bordón, 56 (3 y 4), pp. 409-429.

https://www.researchgate.net/publication/39214322_Viejos_y_nuevos_modelos_de_educacion_a distancia

García-Aretio, L. (2018). Blended learning y la convergencia entre la educación presencial y a distancia. Revista Iberoamericana de Educación a Distancia (RIED), 21 (1), pp. 09-22. https://doi.org/10.5944/ried.21.1.19683

Gómez-Escalonilla, G.; Santín-Durán, M. \& Mathieu, G. (2011). La educación universitaria online en el Periodismo desde la visión del estudiante. Comunicar, 37, pp. 73-80. https://doi.org/10.3916/C37-2011-02-07 
González, M.; Marco, E. \& Medina, T. (2020). Informe de iniciativas y herramientas de evaluación online universitaria en el contexto del Covid-19. Ministerio de Universidades. https://www.uned.es/universidad/inicio/uned_uoc_solidaria.html

Guillén-Gámez, F.D.; Mayorga-Fernández, M.J. (2020) Identification of variables that predict teachers' attitudes towards ICT in Higher Education for teaching and research: A study with regression. Sustainability, 12 (4), pp. 1-14. https://doi.org/10.3390/su12041312

Haug, G. E. (2015). Grados y másteres en España y el espacio europeo de educación superior (EEES). CRUE.

Hernández-Armenteros, J. \& Peréz, J.A. (2019). La universidad española en cifras. Año 2017 y curso académico 2017/2018. CRUE.

Hernández-Ramos, J. P. (2019). Impacto de la brecha generacional digital en la docencia universitaria. En E. M. Fernández Díaz, C. Rodríguez-Hoyos, \& A. Calvo (Eds.), Activismo y tecnología: hacia una universidad comprometida con la educación crítica y emancipadora pp. 212-217. Editorial de la Universidad de Cantabria.

Ibercampus (2020, 12 de mayo). El 60\% de docentes universitarios prefiere clases presenciales tras ver positivas las dadas online. Ibercampus.

https://www.ibercampus.es/el-60-de-docentes-universitarios-prefiere-clases-presenciales-tras-verpositivas-39584.htm

LeBlanc, P. (2020, 16 de marzo). The Coronavirus: Is This Higher Education's Black Swan Event?, Forbes. $\quad$ https://www.forbes.com/sites/paulleblanc/2020/03/16/the-coronavirus-is-this-highereducations-black-swan-event/\#9dc35bcc69a8

Liesa-Orús, M., Latorre-Cosculluela, C., Vázquez-Toledo, S., \& Sierra-Sánchez, V. (2020). The technological challenge facing higher education professors: Perceptions of ICT tools for developing 21st century skills. Sustainability, 12 (13). https://doi.org/10.3390/su12135339

Llorent, M.; Tallon, S.; \& De las Heras, B. (2020). Use of Information and Communication Technologies (ICTs) in Communication and Collaboration: A Comparative Study between University Students from Spain and Italy. Sustainability, 12 (10). https://doi.org/10.3390/su12103969

Martínez, A. (2006). Preguntas de Respuesta Múltiple con SPSS. Curso de SPSS en Español (curso online). http://www.spssfree.com/curso-de-spss/multiples-variables-spss/preguntas-de-respuestamultiple-en-spss.html

Mercader, C. (2020). Explanatory model of barriers to integration of digital technologies in higher education institutions. Education and Information Technologies. https://doi.org/10.1007/s10639$\underline{020-10222-3}$

Ministerio de Universidades (2020). Recomendaciones sobre criterios generales para la adaptación del sistema universitario español ante la pandemia del Covid-19, durante el curso 2019-2020. Conferencia General de

Política

Universitaria. 
RLCS, Revista Latina de Comunicación Social, 78, 265-287

[Investigación] DOI: 10.4185/RLCS-2020-1477| ISSN 1138-5820| Año 2020

https://www.ciencia.gob.es/stfls/MICINN/Universidades/Ficheros/Recomendaciones_adaptacion universidades_CGPU.pdf

Nuere, S., de Miguel, L. (2020). The Digital/Technological Connection with COVID-19: An Unprecedented Challenge in University Teaching. Tech Know Learn. https://doi.org/10.1007/s10758-020-09454-6

Onyema, E. M. et al. (2020). Impact of Coronavirus Pandemic on Education. Journal of Education and Practice, 11 (13), pp. 108-121. DOI: https://doi.org/10.7176/JEP/11-13-12

Orihuela, J. L. (2020, 10 de abril). Repensar la universidad en cuarentena. Medium. https://medium.com/@jlori/repensar-la-universidad-en-cuarentena-34f4d96200bc

Pardo-Kuklinski, H. \& Cobo, C. (2020). Expandir la universidad más allá de la enseñanza remota de emergencia Ideas hacia un modelo híbrido post-pandemia. Outliers School. http://outliersschool.net/project/universidadpostpandemia/

Postigo, I. \& Recoder, M. J. (Eds.) (2018). Los y las tics en los estudios de comunicación. Safekat.

Real Decreto 463/2020, de 14 de marzo, por el que se declara el estado de alarma para la gestión de la situación de crisis sanitaria ocasionada por el COVID-19. https://www.boe.es/boe/dias/2020/03/14/pdfs/BOE-A-2020-3692.pdf

Santín-Durán, M. (2009, 20 de octubre). La enseñanza on-line en el ámbito de la comunicación en España [ponencia]. Felafacs: XIII Encuentro Latinoamericano de Facultades de Comunicación Social. La Habana, Cuba.

Santín-Durán, M.; Mathieu, G. \& Gómez-Escalonilla, G. (2011). Las nuevas tecnologías en la educación: la enseñanza virtual en ciencias de la comunicación en España. En Anuario Electrónico de Estudios en Comunicación Social "Disertaciones", 4 (2), Artículo 2. http://erevistas.saber.ula.ve/index.php/Disertaciones/

Serrano Angulo, J. (2013). Respuestas múltiples en la investigación educativa: codificación, tabulación y análisis. Revista de Investigación Educativa, 31(2), pp. 361-374. https://revistas.um.es/rie/article/view/164111/15813

UNESCO (2020a). Distance learning solutions. https://en.unesco.org/covid19/educationresponse/solutions

UNESCO (2020b, 7 de mayo). Las universidades abordan el impacto de COVID-19 en los estudiantes desfavorecidos. https://es.unesco.org/news/universidades-abordan-impacto-covid-19$\underline{\text { estudiantes-desfavorecidos }}$

UNESCO (2020c) National education responses to COVID-19: summary report of UNESCO's online survey https://unesdoc.unesco.org/ark:/48223/pf0000373322 


\section{AUTOR/ES:}

\section{Antonio J. Baladrón Pazos}

Doctor en Publicidad y RR.PP., Licenciado en Periodismo por la Universidad Pontificia de Salamanca y Licenciado en Publicidad y RR.PP. por la Universidad de Vigo. Es en la actualidad profesor titular de esta especialidad en la Universidad Rey Juan Carlos y coordinador del Grado en Publicidad y RR.PP. de dicha universidad. Ha sido premio extraordinario de doctorado por la Universidad de Vigo y Premio a la Investigación sobre Comunicación de Masas del Consell de l'Audiovisual de Cataluña. Cuenta con más de veinte años de trayectoria docente e investigadora, siendo una de sus líneas de investigación durante los últimos años la metainvestigación pubicitaria. Ha participado en actividades académicas y como profesor invitado en diferentes universidades extranjeras, tanto europeas como americanas.

antonio.baladron@urjc.es

Índice H: 10

Orcid ID: http://orcid.org/0000-0001-7870-2545

Google Scholar: https://scholar.google.es/citations?hl=es\&pli=1\&user=9BrOauQAAAAJ

\section{Beatriz Correyero Ruiz}

Doctora en Ciencias de la Información por la Universidad Complutense de Madrid (UCM) y especialista en Producción radiofónica. En la actualidad es Vicedecana del Grado en Periodismo de la Universidad Católica de Murcia (UCAM). Cuenta con más de quince años de trayectoria docente e investigadora. Sus líneas de investigación son la formación e innovación docente en comunicación, la comunicación radiofónica, el periodismo digital, el análisis bibliométrico y la propaganda de Estado a través del turismo. En la actualidad imparte las asignaturas de Taller de Periodismo Multimedia e Información en Radio en el Grado en periodismo de la UCAM y es la coordinadora de prácticas externas de la titulación.

bcorreyero@ucam.edu

Índice H: 10

Orcid ID: http://orcid.org/0000-0003-0069-8448

Google Scholar: https://scholar.google.es/citations?user=cB4A-6sAAAAJ\&hl=es

ResearchGate: https://www.researchgate.net/profile/Beatriz_Correyero_Ruiz

Scopus ID: $\underline{\text { https://www.scopus.com/authid/detail.uri?authorId }=55123230200}$

\section{Benjamín Manchado Pérez}

Doctor por la Universidad Complutense de Madrid, en el Departamento de Estadística e Investigación Operativa II (Métodos de Decisión). Es coautor de varios artículos científicos, tratando temas como la investigación publicitaria utilizando técnicas bibliométricas y de análisis multivariante, o el fraude académico en la universidad española. Posee una experiencia profesional de más de veinte años en puestos de responsabilidad en el área financiera de empresas de distintos sectores: banca, gestión de patrimonios, construcción, sanidad, agroalimentario; así como varios años de docencia universitaria de grado. En la actualidad compagina su posición de Responsable de Market Intelligence del Grupo Agroponiente en Almería con la labor de docencia en el MBA en inglés que imparte la Universidad Católica San Antonio de Murcia.

benjaman@ucm.es

Índice $\mathrm{H}: 5$

Orcid ID: http://orcid.org/0000-0001-9176-3182

Google Scholar: https://scholar.google.es/citations?user=wC-PsMIAAAAJ\&hl=es

ResearchGate: https://www.researchgate.net/profile/Benjamin_Manchado 